

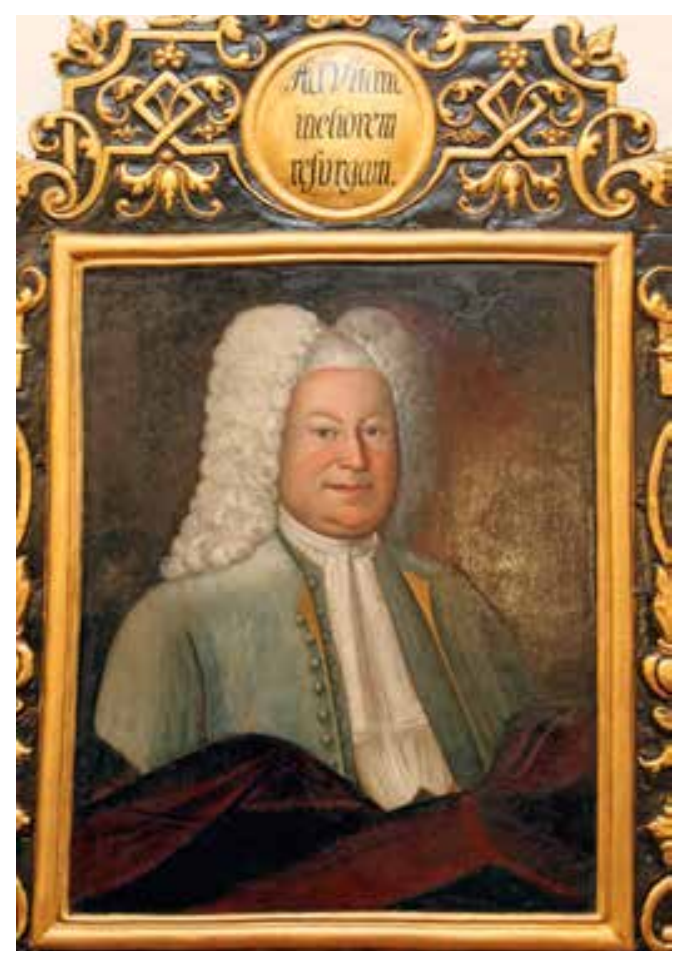

Durch glückliche Umstände ist uns dieser Text im Ratsarchiv ebenso erhalten geblieben wie die Einträge in den Kirchenbüchern seit 1608. Vom ersten Standort der Apotheke, dem verlassenen Franziskanerkloster, ist allerdings nichts mehr erhalten. Indessen geben die Archive auch über die Folgezeit Auskunft.

\section{Nachfolgende Apotheker}

Auf Johann Seiler (1611-1626) folgten Georg Griffner (1626-1666) und Dr. Johann Zeidler (1666-1672), der die Apotheke 1666 in die Nicolaistraße 4 verlegte.

Anna Maria Zeidler (1677-1690) ließ die Apotheke bis zum Eintritt ihre Sohnes Conrad Zeidler von Rosenberg (1690-1733) vermutlich durch einen angestellten Apotheker betreiben. Conrad Zeidler war gleichzeitig Bürgermeister und brachte es als erster Löbauer Apotheker zu Wohlstand. Sein Bild ist uns auf einem opulenten Epitaph erhalten, wie es für alle Bürgermeister angefertigt wurde.

Nachfolger als Apotheker waren: Dr. Gotthard Zeidler von Rosenberg (1731-1733), Johann Friedrich Zink (1733-1742), Christian Friedrich Plato (1742-1769), Carl Christian Kielmann (1769-1801) und Carl Benjamin Kanig (1801-1821). Letzterer verlegte die Apotheke in die Kirchgasse 4, die 1886 in Bahnhofstraße 2 umbenannt wurde. Es folgten Johann Eisler (1821-1822), Christoph Friedrich Salzmann (1822-1844) und Johann Heinrich Brückner (1844-1877).

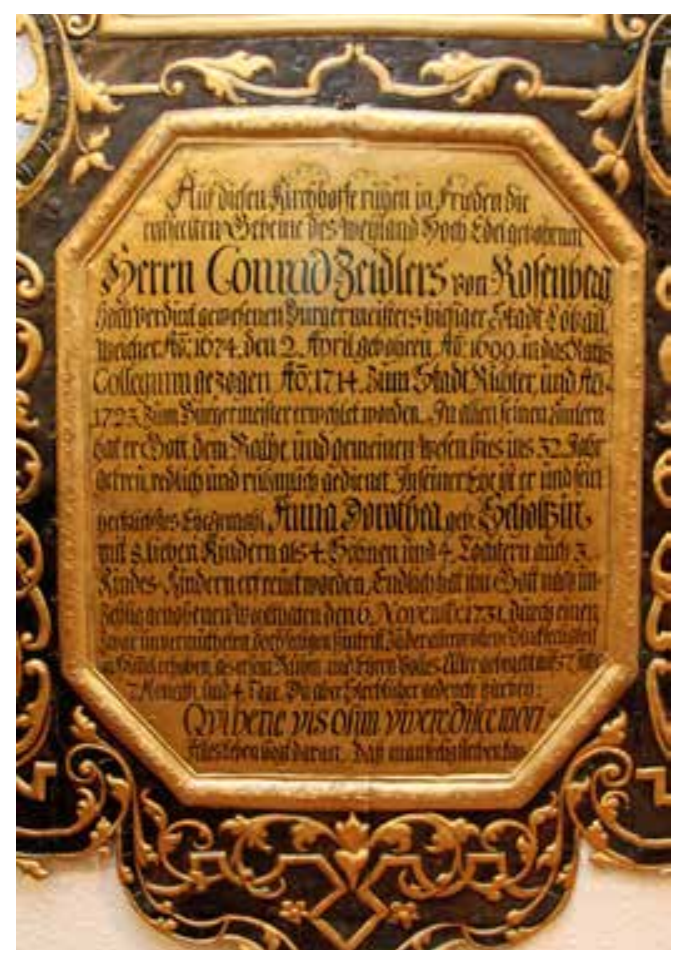

Epitaph für Conrad Zeidler von Rosenberg, Ausschnitte

Die Konkurrenz kündigt sich an

Johann Heinrich Brückner, aus Kamenz stammend, war zielstrebig tätig. Er lebte sparsam und war in der Stadt sehr angesehen. Sein Sohn Heinrich Curt Brückner (1877-1917) hatte es eilig und scheute keine Mühe, aus der Apotheke einen Musterbetrieb zu machen. Der Grund war naheliegend, denn schon vor 1900 drängten aufgrund der Gewerbefreiheit junge Apotheker in die Selbständigkeit und somit auch nach Löbau. Als Stadtverordneter und später

Ältestes Bild der Löbauer Apotheke, um 1860

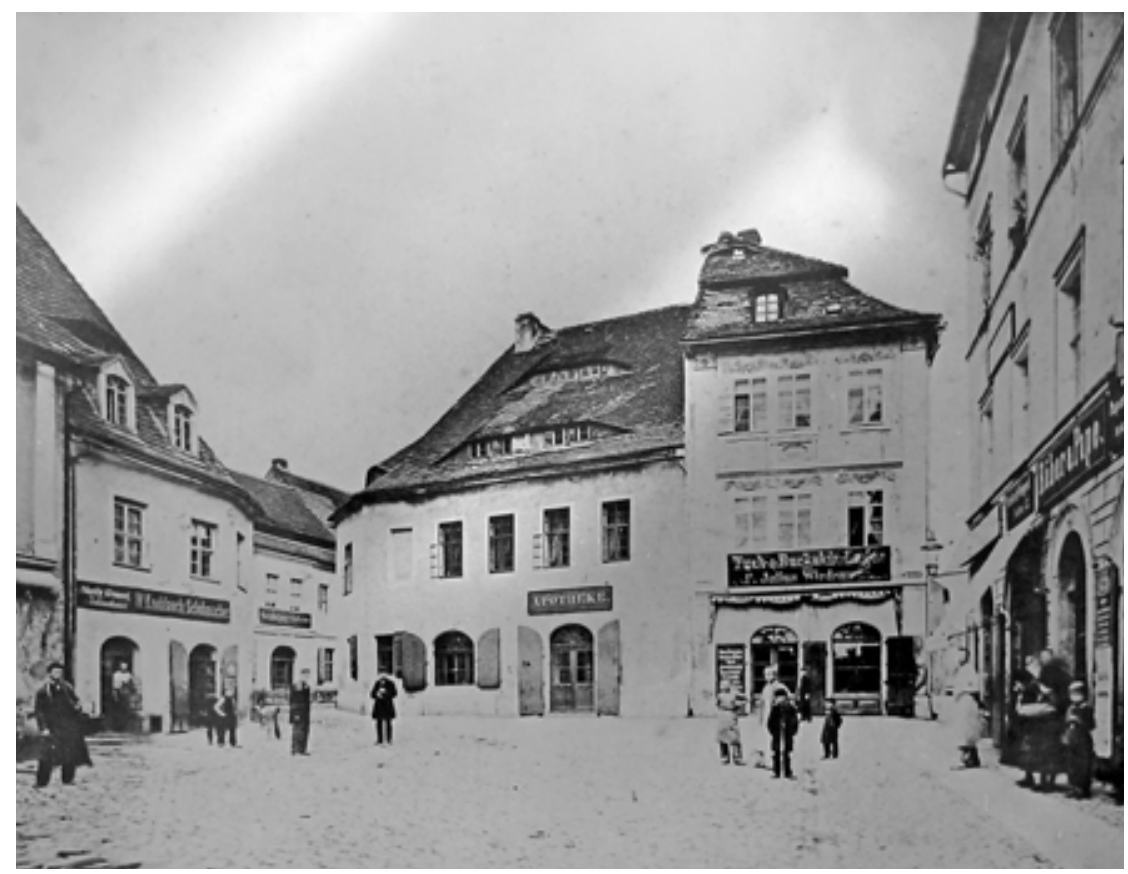


stellvertretender Bürgermeister konnte Curt Brückner lange Zeit die Konkurrenz verhindern, aber am 1. November 1909 eröffnete Oskar Anton Kammerlander im Gebäude Innere Zittauer Straße 12 die Johannis-Apotheke.

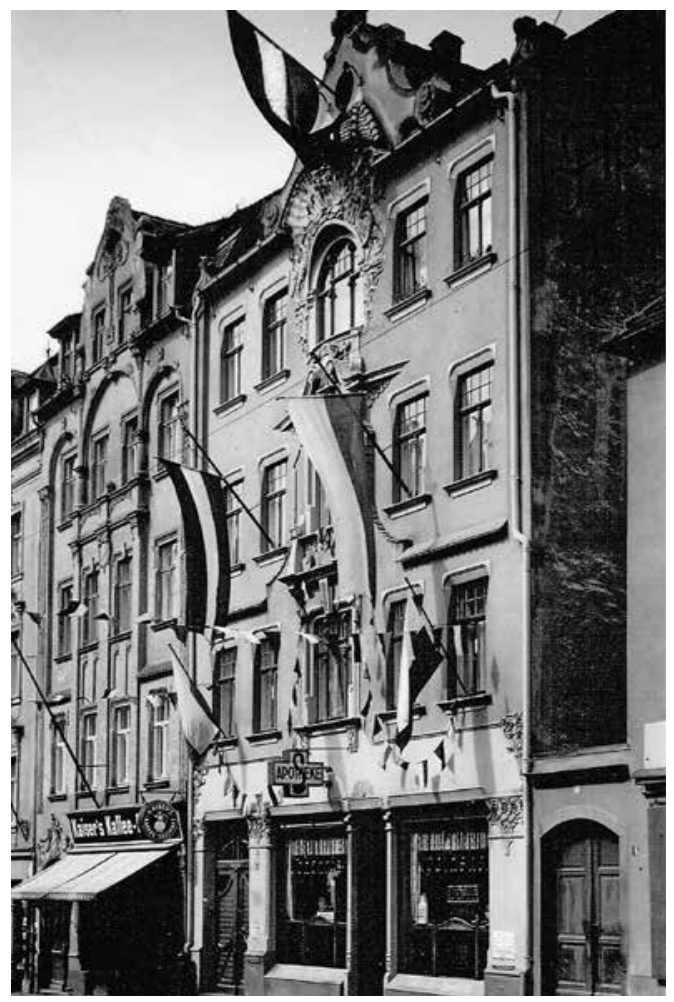

Die Vergabe eines Privilegs war damals schon abgeschafft. Stattdessen war eine sogenannte Personalkonzession ausschließlich für den Inhaber gültig. Somit war die Apotheke - genauer gesagt die Konzession - weder verkäuflich noch vererbbar, denn diese fiel im Todesfalle an den Staat zurück. Auf Oskar Anton Kammerlander (1909-1919) folgten Dr. phil. Johannes Maximilian Heinze (1920-1921), Paul Robert Hugo Thomann (1921-1940), Hans Lex (19401958). Während dessen Dienstzeit erfolgte 1953 die Verstaatlichung der Johannis-Apotheke. Er blieb bis 1958 staatlich angestellter Leiter. Nachfolgerin wurde Charlotte Drechsel (1958-1962) und zuletzt Käthe Schurig (19631966).

\section{Curt Brückners herausragende Verdienste}

Wir begeben uns noch einmal zurück in das Jahr 1909 zu Curt Brückner. Er machte in diesem Jahr seinen Sohn Curt Erich Brückner zum Teilhaber und $1917 \mathrm{zu}$ seinem Nachfolger. Bis zu seinem Tod im Jahr 1922 erhielt Curt Brückner für seine Verdienste um die Stadt Löbau sowie um den Berufsstand in der Oberlausitz viele Ehrungen, so 1909 das Ritterkreuz I. Klasse,
1914 die Ernennung zum Königlich Sächsischen Hofrat und schließlich 1921 die Ehrenbürgerschaft der Stadt Löbau.

Die bereits 1911 erfolgte Umbenennung der Theatergasse in „Brücknerring“ hat bisher über alle politischen Systeme hinweg Bestand.

\section{Die Nachfolger von 1917 \\ bis zur Gegenwart}

Curt Erich Brückner führte die Apotheke von 1917 bis 1956, nach der Verstaatlichung vom 1. Oktober 1954 als vom Staat angestellter Apothekenleiter. In die Dienstzeit seines Nachfolgers Gerhard Hellriegel (1956-1968) fiel eine weitreichende Rekonstruktion der Apotheke. Da diese tief in die Gebäudesubstanz eingriff, wurde die Apotheke für eineinhalb Jahre vom 1. September 1964 bis zum 28. Februar 1966 geschlossen. Die Versorgung der Löbauer Bürger erfolgte in dieser Zeit ausschließlich durch die Johannis-Apotheke, die mit der Wiedereröffnung der Alten Apotheke am 1. März 1966 ihren Betrieb einstellte.

Der Umbau umfasste zunächst nur den vorderen Abschnitt des Erdgeschosses und die technische Gebäudeausrüstung. Wieland Schäfer, seit 1968 mit der Leitung der Alten Apotheke beauftragt, arbeitete bis 1989 schrittweise an der Fortsetzung des Ausbaus der Einrichtung, die auch viele Aufgaben für den Kreis Löbau wahrnahm. Bis zur Wende war in zwei Etagen ein moderner Betrieb entstanden, der zu den größten öffentlichen Apotheken des Bezirkes Dresden zählte.

\section{0 beginnt für die Apotheken eine neue Ära}

Die Wende brachte in allen Bereichen Veränderungen, die eine Angleichung an das in den Altbundesländern geltende System zum Ziel hatte. In der Bundesrepublik Deutschland gab es kein staatliches Apothekenwesen. Alle öffentlichen Apotheken waren inhabergeführt und befanden sich im persönlichen Eigentum des leitenden Apothekers.

\section{Veränderungen in der Alten Apotheke}

Bereits zum Zeitpunkt des Kaufs der Staatlichen Alten Apotheke von der Treuhandgesellschaft am 15. September 1990 war für Wieland Schäfer klar, dass neben den Auswirkungen der Währungsunion vom 1. Juli 1990 weitere Veränderungen bevorstehen. Schon im Juli 1990 flossen Warenströme aus den Altbundesländern in Größenordnungen in die noch beste- 
hende DDR und verdrängten einheimische Produkte. Damit war die umfangreiche Eigenherstellung von Arzneimitteln ebenso überflüssig geworden wie die über die Kreisgrenzen hinaus gefragte zentrale Tabletten- und Augentropfenproduktion. Gleichzeitig gingen traditionelle Kunden verloren. Von den eben noch 67 Abnehmern im staatlichen Gesundheitswesen (u. a. Krankenhaus für Gynäkologie und Geburtshilfe, Betriebssanitätsstellen, Kinderkrippen, staatl. Arzt- und Zahnarztpraxen) existierte nach einigen Monaten nur noch ein kleiner Teil. Dadurch verlor die Hälfte der 40 Mitarbeiterinnen der Alten Apotheke ihren Arbeitsplatz. Ein Teil von ihnen wurde aber bald wieder an anderer Stelle gebraucht.

Die Johannis-Apotheke wird wieder aktiv

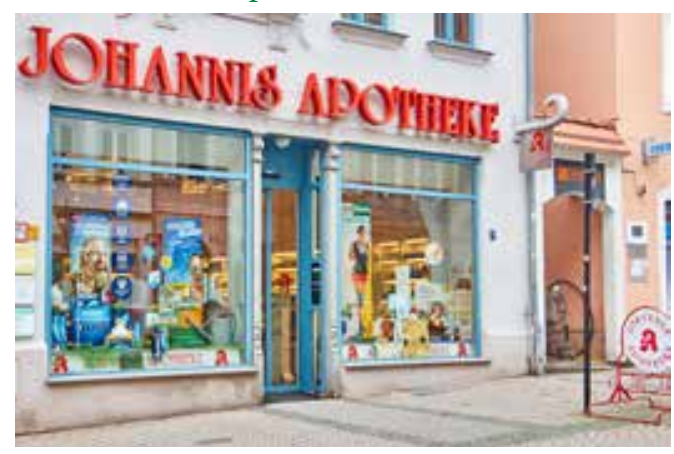

Im Dezember 1990 löste sich die staatliche Handelsorganisation (HO) auf. Damit stellte auch das Sportartikelgeschäft, das sich in den Räumen der 1966 geschlossenen Johannis-Apotheke befand, seinen Betrieb ein. Apotheker Dr. Hartmut Scheibner sah die Chance, die seit 24 Jahren geschlossene Johannis-Apotheke zu reaktivieren. Die Nutzungsgenehmigung der Gebäudewirtschaft gab ihm die Möglichkeit, bereits im Frühjahr 1991 die Instandsetzung des Gebäudes und die Einrichtung einer Apotheke nach modernen Gesichtspunkten vorzunehmen. Wenn sich auch der Erwerb des Gebäudes bis 1995 hinzog, konnte die Eröffnung am 17. September 1991 stattfinden. Die „neue“ Johannis-Apotheke etablierte sich rasch, denn sie war ja vielen Löbauern noch in Erinnerung.

\section{In der Löbauer Neustadt eröffnet die Linden-Apotheke}

Schon zu DDR-Zeiten bestand der dringende Wunsch, in der Löbauer Neustadt - im Stadtteil nördlich der Bahngleise - eine Apotheke zu haben, zumal sich dort auch die Polikliniken sowie mehrere Arzt- und Zahnarztpraxen befanden. Als ein Investor aus Offenbach nach der Wende das Werksgelände der ehemaligen Frottierwa-

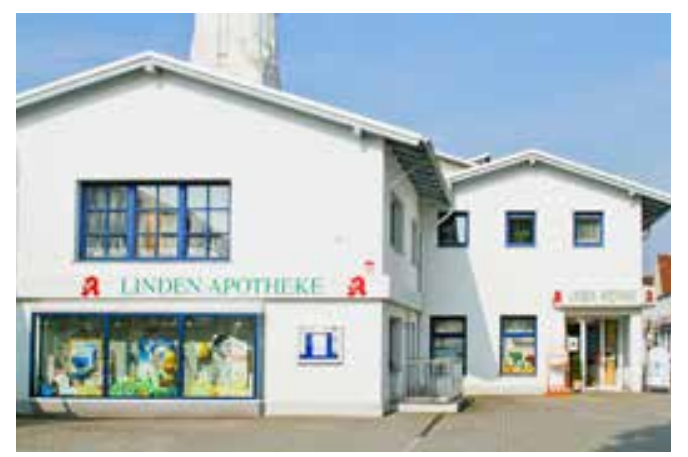

Linden-Apotheke

renfabrik Rönsch \& Söhne erwarb, um ein großes Einkaufszentrum zu errichten, war der Umbau der Betriebsschlosserei zu einer Apotheke vorgesehen. Apothekerin Ursula Schäfer bewarb sich und erhielt den Zuschlag für die neu entstehende Apotheke. Als der Gewerbepark Ende Januar 1991 öffnete, waren in der neuen Linden-Apotheke noch Bauverzögerungen $\mathrm{zu}$ meistern, so dass sich die Eröffnung auf den 29. März 1992 verschob. Damit war ein lang gehegter Wunsch der Neustadtbewohner in Erfüllung gegangen. Das Profil der Apotheke wurde über lange Jahre durch die benachbarte Hautarztpraxis geprägt. Die räumliche Enge konnte durch die Einbeziehung einer benachbarten Gewerbeeinheit behoben werden. Seit 2009 gehört die Linden-Apotheke als Filiale zu der von Wieland Schäfer und seiner Tochter Birgit Schleicher betriebenen Alte Apotheke OHG.

\section{Aus der Poliklinik wird ein modernes Ärztehaus mit der Aesculap-Apotheke}

Mit der Wende entfielen die Strukturen des staatlichen Gesundheitswesens. Ärzte und Zahnärzte drängten in die eigene Niederlassung, wobei geeignete Räumlichkeiten nicht ausreichend zur Verfügung standen. Alle Hoffnungen richteten sich auf das Großprojekt eines Ärzte- und Zahnärztehauses, das ein Investor aus Lörrach auf freiem Feld in der Neusalzaer Straße plante. Wegen fehlender Grunderwerbsmöglichkeiten gab der Investor 1991 seine Pläne auf.

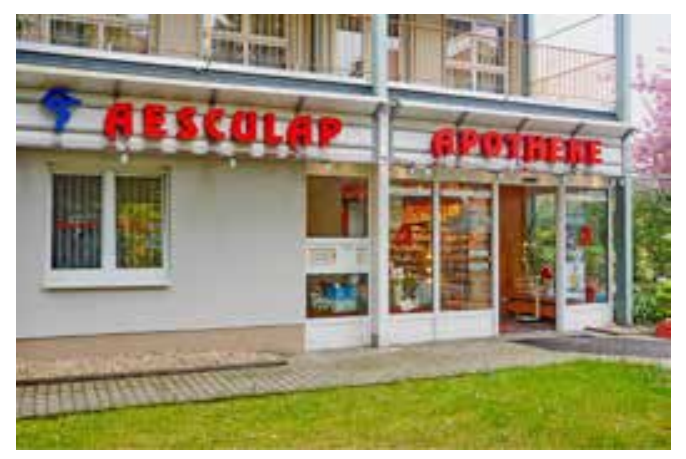

Johannis-Apotheke 
Bahnhof-Apotheke Alte Apotheke

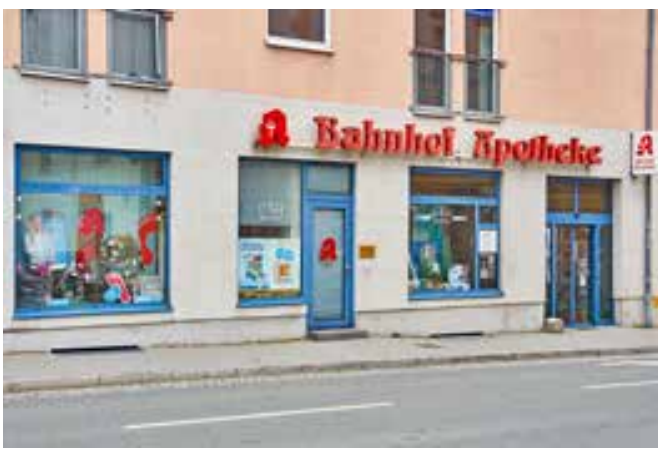

Indessen sahen die Ärzte in der Poliklinik Breitscheidstraße mit Sorge den baulichen Verfall ihres Domizils. Der Mut einer Bauherrengemeinschaft wurde schließlich belohnt. Das 1975 errichtete Gebäude wurde ab Februar 1995 grundlegend renoviert und erhielt eine eigene Apotheke. Die Baumaßnahmen an der großen Außentreppe waren noch im Gange, als Apotheker Michael Thiele am 7. März 1996 seine Aesculap-Apotheke eröffnete. Auch 20 Jahre nach diesem aufwendigen Umbau wird dieses modernisierte Ärztehaus den Anforderungen der heutigen Zeit gerecht.

Eine weitere Apotheke entsteht - die Bahnhof-Apotheke in der Sachsenstraße

Die größte innerstädtische Baustelle befand sich von 1997 bis 1998 in der Sachsenstraße. In der Bauplanungsphase gab es mehrere Nutzungsänderungen. Von dem ursprünglich geplanten größeren Hotel wurde zugunsten von Wohnungen abgesehen. Zum Maklerobjekt gehörte auch die Errichtung einer Apotheke. Daran interessierte Mieter fanden sich im Januar 1998, als das Apothekerehepaar Sabine und Dr. Lutz Hentschel seinen Standort in Zittau aufgab und sich in der Sachsenstraße unter Mitnahme des Apothekennamens wiederum als „BahnhofApotheke“ niederließ. Zum 1. Januar 2004 erwarb der Apotheker Dr. Ralf Krowke aus Zittau die Bahnhof-Apotheke und gliederte sie seiner in Zittau betriebenen Herz-Apotheke als Filialapotheke an. Filialleiter wurde Apotheker Uwe Hoffmann. Als Dr. Ralf Krowke beide Apotheken verkaufte, wurde Uwe Hoffmann am 1. Januar 2006 Inhaber.

Die Alte Apotheke - nach 400 Jahren am Altmarkt angekommen

Im Zeitraum ab 1990 gab es auch in der Alten Apotheke erhebliche Veränderungen. Der grundlegende Umbau der damals noch einzigen Apotheke in Löbau verbot eine zeitweilige Schließung. Im Zeitraum vom März bis Juni

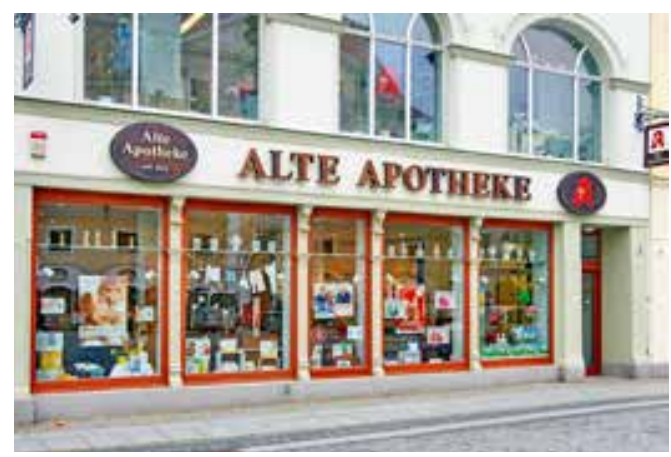

1991 wurde nach sorgfältiger Planung die historische Bausubstanz so verändert, dass am 3. Juli 1991 der Löbauer Bevölkerung eine moderne Apotheke zur Verfügung stand, die jedem Vergleich mit den Altbundesländern standhielt. Mit der Gründung der Alte Apotheke OHG am 1. Juli 2006 wurde die Kraft von zwei Apothekergenerationen gebündelt. Wieland Schäfer und Birgit Schleicher konnten dadurch auch die bisher größten Herausforderungen bestehen - den Umzug der Apotheke in die modernisierten Gebäude Nr. 5 und 6 am Altmarkt, gegenüber dem Rathaus. Es war die dritte Standortveränderung in der 400-jährigen Geschichte der Alten Apotheke. Sie erfolgte im Jubiläumsjahr 2011.

\section{Fünf Apotheken finden ihr eigenes Profil}

Wenn man von den Inhaberwechseln und dem Umzug der Alten Apotheke absieht, hat sich die Apothekenlandschaft in Löbau seit 1998 nicht mehr verändert. Im Rahmen des gemeinsamen

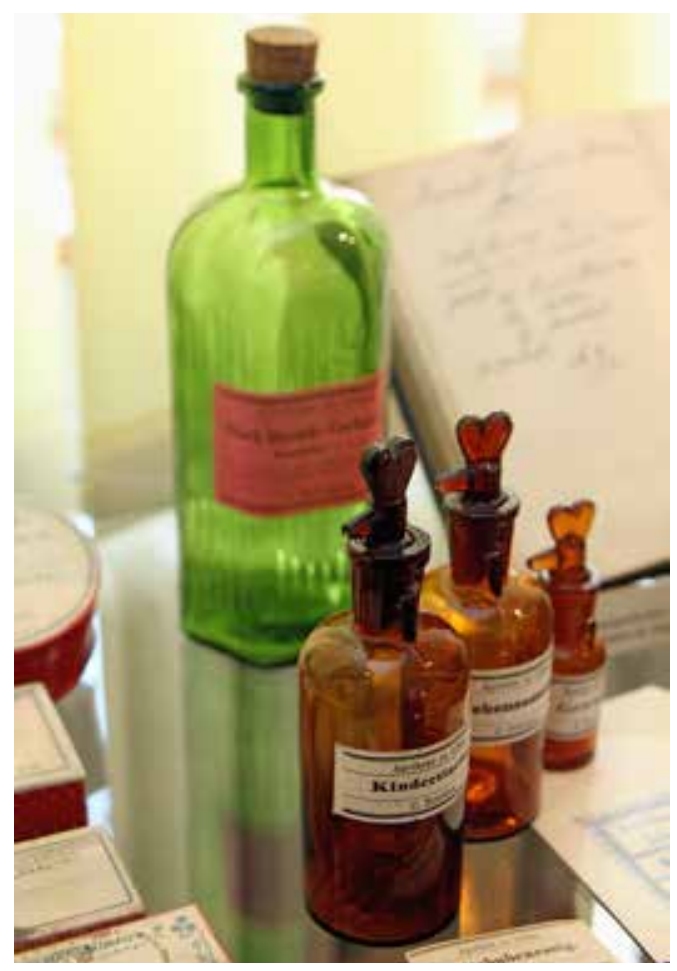



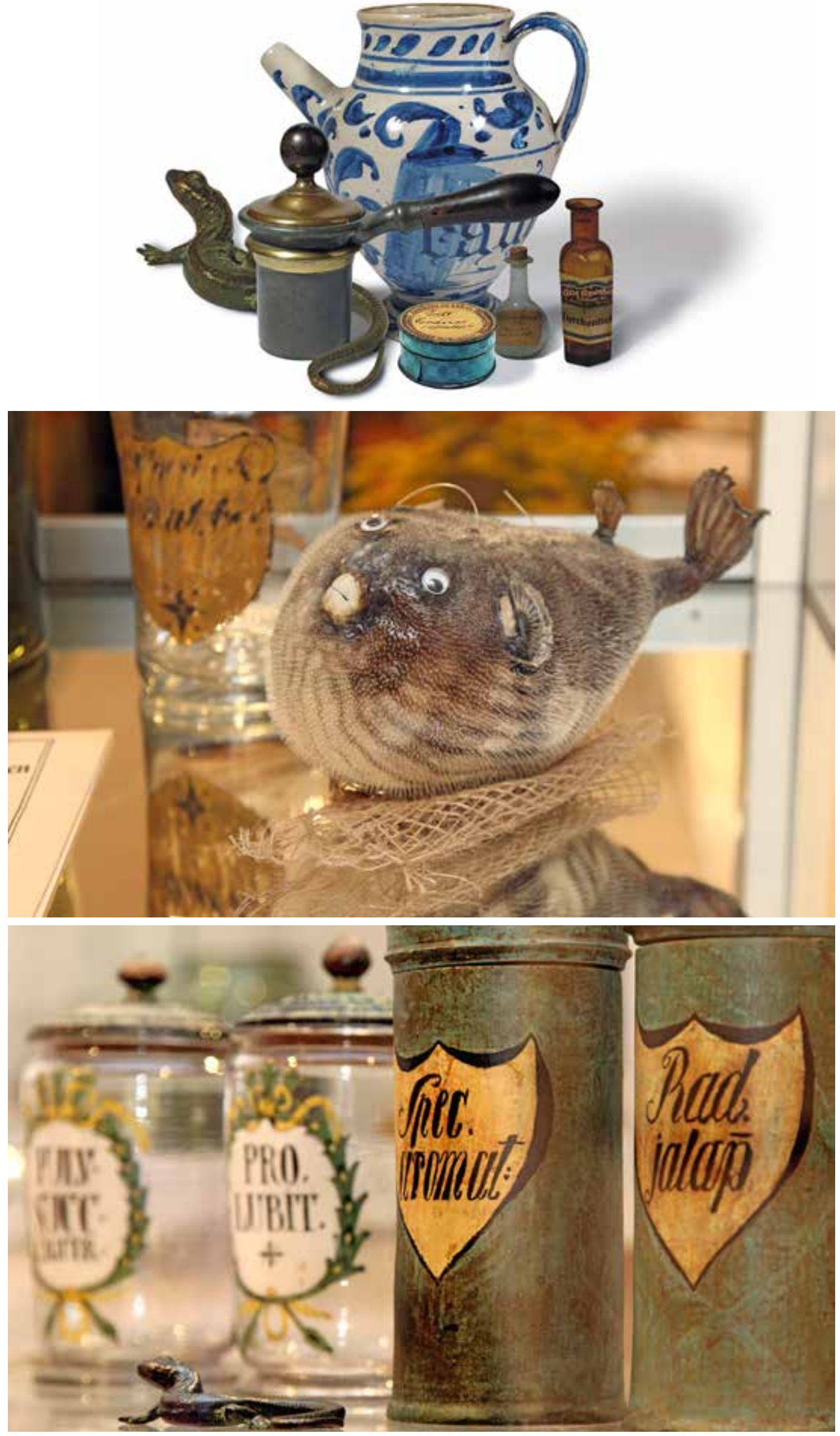
Jubiläumsmedaille 2011

Quellen Petra Ulbricht: Zur Geschichte des Apothekenwesens der Stadt Löbau. Ungedr. Abschlussarbeit Ingenieurschule für Pharmazie Leipzig, 1982.

Wieland Schäfer: Die Apotheke zu Löbau. Ein Streifzug durch ihre 400-jährige Geschichte, 2011.

Mündliche Auskünfte erteilten Apotheker Dr. Hartmut Scheibner, Apotheker Michael Thiele, Apotheker Uwe Hoffmann

Löbauer Sachsentaggroschen 2017

Autor

Wieland Schäfer Alte Apotheke OHG



Versorgungsauftrags hat jede der fünf Apotheken ihr eigenes Profil entwickelt. Darüber hinaus werden unterschiedliche am Gemeinwohl orientierte Projekte verfolgt.

Die Alte Apotheke nahm ihr 400-jähriges Bestehen zum Anlass, ihre Geschichte umfassend zu erforschen und sowohl für die Fachwelt als auch für interessierte Laien verständlich darzustellen. Die über sechs Monate im Stadtmuseum gezeigte Ausstellung mit ausgewählten Exponaten zur Pharmaziegeschichte fand bundesweit Beachtung. Eine von Dipl.-Designer Klaus Herzog gestaltete Medaille erinnert an die 18 Apotheker, die die Apotheke während der 400 Jahre besaßen bzw. leiteten.

Es war absehbar, dass das Löbauer Stadtmuseum die pharmazie-historische Ausstellung nicht dauerhaft zeigen kann, denn sie nahm das ganze Erdgeschoss in Anspruch. Die Bereitschaft des Eigentümers, die Exponate der Öffentlichkeit dauerhaft zugänglich zu machen, ist weiterhin vorhanden. Bisher wurde jedoch aus eigener Kraft keine Lösung gefunden, so dass Vorschläge oder Angebote vom Autor dieses Beitrages

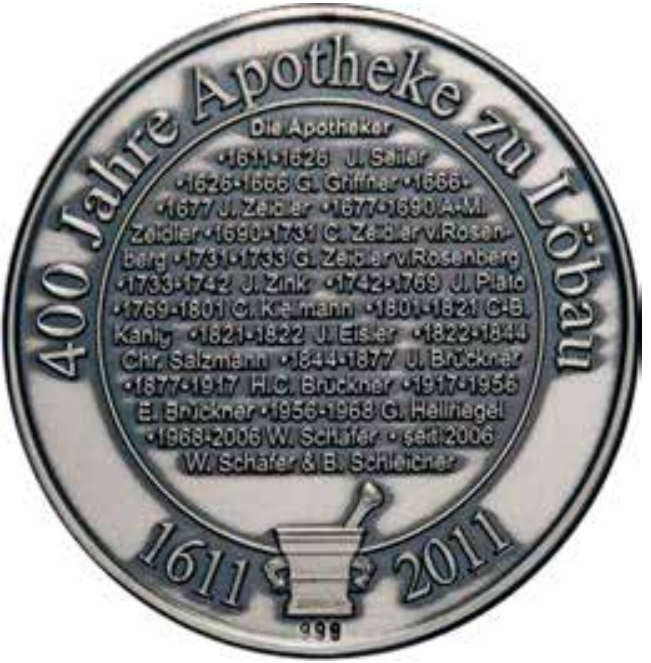

jederzeit dankbar angenommen werden. Anlässlich der Landesgartenschau 2012 in Löbau gestaltete die Alte Apotheke OHG in $\mathrm{Zu}$ sammenarbeit mit dem Landesverband der Sächsischen Kleingärtner e. V. und dessen Territorialverband Löbau einen Apothekergarten, der viel Beachtung fand.

Zum Sächsischen Erntedankfest 2015 wurde gemeinsam mit örtlichen Imkern das Thema „Honig“ in den Blickpunkt gerückt.

Von der Sächsischen Numismatischen Gesellschaft wurde der Alte Apotheke OHG 2016 der Wunsch angetragen, für die Gestaltung und Herstellung des sogenannten Auswurfgroschens zu sorgen. Für die Gestaltung der Rückseite der von Klaus Herzog entworfenen Medaille wurde ein Prunkmörser von 1635 ausgewählt. Der Mörser als ältestes Arbeitsgerät des Apothekers, das heute noch in Gebrauch ist, soll die Brücke von der langen Tradition des Berufstandes zur Gegenwart bilden und dazu beitragen, sich in Verbindung mit der von Lars-Gunter Schier gestalteten großen Gedenkmedaille auch später noch an den Löbauer Tag der Sachsen zu erinnern.
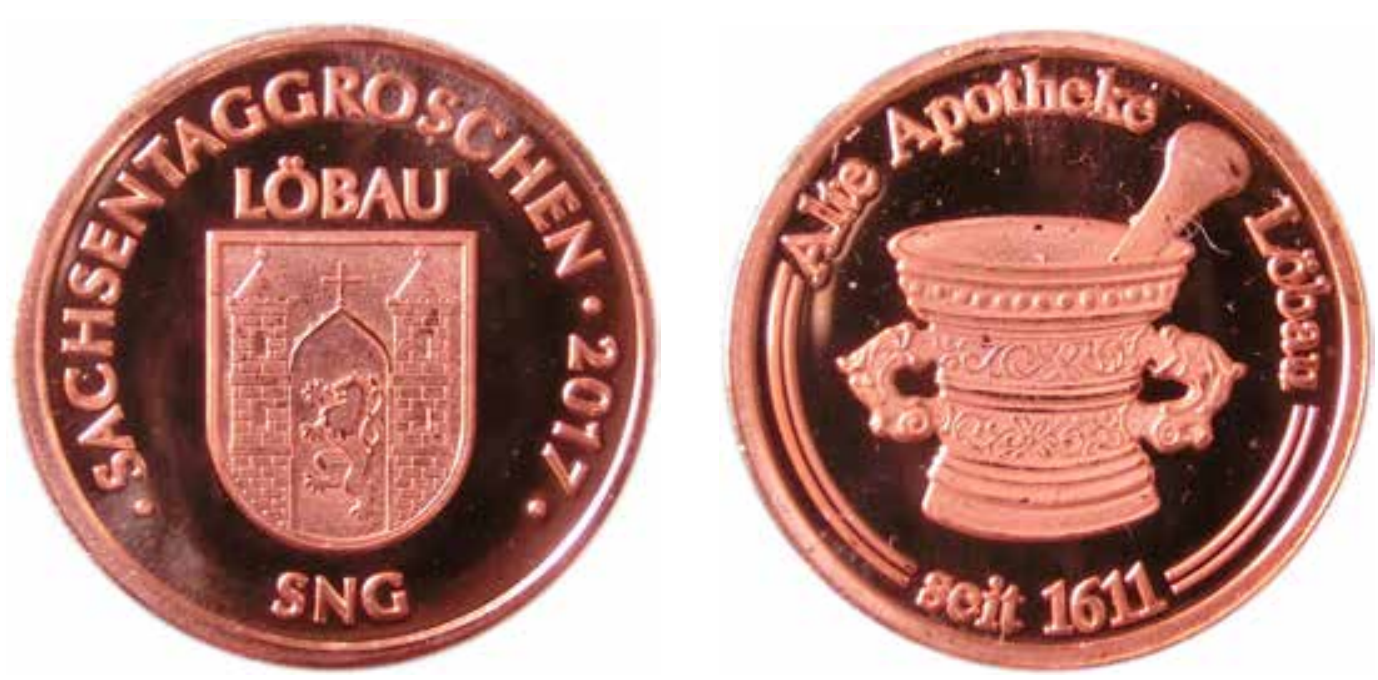